\section{Middle Cerebral Artery}

Elliot J. Roth

Northwestern University, Feinberg School of Medicine, Physical Medicine and Rehabilitation, Chicago, IL, USA

\section{Synonyms}

Cerebral artery; MCA

\section{Definition}

The middle cerebral artery (MCA) arises from the internal carotid artery, ascends into brain where it provides blood supply to most of the lateral cerebral cortex, anterior temporal lobes, insular cortex, and part of the internal capsule and basal ganglia. It has branches in the frontal, parietal, and temporal lobes.

\section{Current Knowledge}

The MCA is divided into four segments: The M1 or sphenoidal segment is usually horizontal and tracks the sphenoid bone providing numerous perforating anterolateral central (lateral lenticulostriate) arteries, which perfuse the basal ganglia. The M2 or insular segment extends branches toward the cortex. The M3 or opercular segment extends laterally toward the cortex. The M4 or cortical segment begins at the Sylvian fissure and perfuse the cortex.

Occlusion of the middle cerebral artery constitutes the most common and significant mechanism and location of strokes. These strokes may result in contralateral hemiplegia of the face and limbs (hemiplegia), hemisensory loss in the contralateral face and limbs, homonymous hemianopsia or quadrantanopia, aphasia when the dominant hemisphere is affected, and hemiinattention and other perceptual deficits when the nondominant hemisphere is affected.

\section{Cross-References}

$>$ Circle of Willis

- Internal Carotid Artery

$\checkmark$ Ischemic Stroke 